\title{
ARTICLE
}

\section{A comparison of DSM and ICD classifications of mental disorder}

\author{
Peter Tyrer
}

Peter Tyrer is Professor of Community Psychiatry in the Centre for Mental Health in the Division of Medicine at Imperial College London. His main interests are in models of delivering community psychiatric services, the classification and treatment of common mental illnesses, particularly anxiety and health anxiety, and the classification and management of personality disorders Correspondence Professor Peter Tyrer, Centre for Mental Health, Department of Medicine, Imperial College London, St Dunstan's Road, London W6 8RP, UK. Email: p.tyrer@ imperial.ac.uk

\begin{abstract}
SUMMARY
Most disorders in medicine are classified using the ICD (initiated in Paris in 1900). Mental and behavioural disorders are classified using the DSM (DSM-I was published in the USA in 1952), but it was not until DSM-III in 1980 that it became a major player. Its success was largely influenced by Robert Spitzer, who welded its disparate elements, and Melvyn Shabsin, who facilitated its acceptance. Spitzer pointed out that most diagnostic conditions in psychiatry were poorly defined, showed poor reliability in test-retest situations, and were temporally unstable. The consequence was that the beliefs of the psychiatrist seemed to matter much more than the characteristics of the patient when it came to classification. Since DSM-III there has been a split between those who adhere to DSM because it is a better research classification and those who adhere to ICD because it allows more clinical discretion in making diagnoses. This article discusses the pros and cons of both systems, and the major criticisms that have been levelled against them.
\end{abstract}

\section{LEARNING OBJECTIVES}

- Understand the principles and reasoning behind classification in medicine and psychiatry.

- Be able to describe the recent history of psychiatric classification.

- Be able to compare DSM and ICD classifications of mental disorder.

\section{DECLARATION OF INTEREST}

P.T. is Chair of the ICD-11 Working Group for the Revision of Classification of Personality Disorders and has also been a member of the DSM-ICD Harmonization Coordination Group.

Although diagnosis in psychiatry leads to a lot of argument - and the extent of this dissatisfaction is expressed by an international campaign initiated by Sami Timimi (2011) on the Critical Psychiatry Network UK to abolish the ICD and DSM classifications altogether - most psychiatrists regard classification as having some value. A good classification provides information about the causes of disorder, possible prevention, clinical characteristics, natural history (i.e. the course of the disorder without intervention), and a guide to treatment and outcome. Without a classification system the necessary economical communication with colleagues to convey information becomes a lengthy description of clinical problems that is self-defeating.

The difficulties of classification in psychiatry are many and the reasons why we have arguments on the subject and why others in medicine do not to the same degree is summarised in Box 1.

\section{Biological markers}

The aim of psychiatric classification for many years has been to find an independent set of biological or pathophysiological markers that indicate the presence of disease and can therefore confirm clinical impressions. This is a perfectly natural aim, as it is following in the footsteps of medicine, where almost every disorder receives respectability from having such an independent biological basis. Nobody is disputing that every disorder in psychiatry has some sort of biological associates, but for most of them it is of no value in diagnosis because our current knowledge is inadequate. The problem is mainly lack of specificity and impaired understanding of the fundamental biological processes involved in much of mental disorder.

BOX 1 Differences between diagnosis of psychiatric and medical disorders

Psychiatric disorders

- Biology: very few psychiatric disorders have a biological basis that confirms clinical impressions of disease

- 'Zone of rarity' between health and disease rarely present in psychiatric disorders

- Uncertain threshold of diagnosis

Medical disorders

- Most diagnoses in medicine have a biological basis that can confirm disease (e.g. blood pressure in hypertension, blood sugar in diabetes)

- 'Zone of rarity' between health and disease more often, but not always, present

- Better thresholds for diagnosis (usually because independent tests can confirm clinical impressions) 
To take one example among many, it has been found that many individuals with obsessivecompulsive disorder (OCD) have post-streptococcal autoimmunity autoantibodies related to possible infection in childhood (Dale 2005). In this study, $42 \%$ of children with OCD had these autoantibodies, but no one would suggest that testing for autoantibodies would be a suitable diagnostic test for OCD, not least as up to $10 \%$ of controls also had these autoantibodies.

The same does not apply in medicine for most disorders. If you develop lobar pneumonia, X-rays will confirm that you have consolidation in your lung and analysis of the sputum will confirm that you have a pneumococcal infection. When identified, these abnormalities are indisputable, and the clinical examination of the chest that leads towards the diagnosis of lobar pneumonia is only a preliminary diagnostic exercise that remains to be confirmed by the X-ray and sputum analysis.

In psychiatry, we do not have the insight of independent tests for most of our disorders, but this does not mean they do not exist. We do have independent measures for conditions such as Alzheimer's disease and Down syndrome, but these are rare compared with more common conditions such as anxiety, depression and personality disorder. Readers will note from looking at psychiatric journals that there are increasing numbers of neuroanatomical and imaging studies that find abnormalities in people with different psychiatric disorders, but in none of these is the abnormality clearly diagnostic (i.e. it occurs in every person with the disorder and is absent in those who do not have the disorder).

As a consequence of this lack of independent evidence, there are many more models of mental disorder than models of physical disorder (Tyrer 2013). This explains a lot of the controversy that exists within psychiatry - controversy that is generally lacking in medicine but still emerges when trying to find diagnostic cut-off points for the treatment of conditions such as hypertension and metabolic syndrome. Unfortunately, the presence of many different models still includes an antipsychiatry one that abhors diagnosis altogether, with only the disease of labelling being allowed to show itself. But this notion has to be put to rest; psychiatry without diagnosis will return us to the Dark Ages and has to be embraced in some form (Craddock 2014).

\section{Zone of rarity}

Introduced by Robert Kendell (1968), the 'zone of rarity' refers to the hiatus between the features of a biological disorder with a clear diagnosis and other conditions that do not carry this diagnosis.
Take the example illustrated in Fig. 1. Depression is commonly classified by a score on a standard inventory or by the number of criteria needed to cross the threshold into diagnosis. But every clinician and researcher knows that the threshold is not a clean one - there are many people just below the threshold, who have many of the symptoms of depression but who do not quite qualify for diagnosis. There is now abundant research that shows that these people are, not surprisingly, as unwell as others who are just over the threshold line (Ayuso-Mateos 2010).

By contrast, the diagnosis of iron-deficiency and pernicious anaemia depends on analysis of data from red blood cells that are much more specific. There is a zone of rarity between those who have macrocytic anaemia (large corpuscles characteristic of vitamin $\mathrm{B}_{12}$ deficiency and irondeficiency corpuscles) and between those who have normal blood cells (hence the three groupings in Fig. 1). If we just relied on clinical examination, the separation of these anaemias would be much more difficult and might approximate to the data for depression. Because we have no equivalent biomarker for depression, there is no zone of rarity.

It is also relevant that for many chronic medical conditions, such as osteoarthritis, Parkinson's disease and obstructive airways disease, there is no zone of rarity either, so although it is an excellent illustration for disorders such as Huntington's chorea and porphyria, the more common conditions that flesh is heir to do not share this distinction.

\section{Uncertain threshold of diagnosis}

The absence of a zone of rarity indicates that the threshold for psychiatric diagnosis is usually arbitrary. When psychiatrists make a decision about a clinical diagnosis they therefore have no

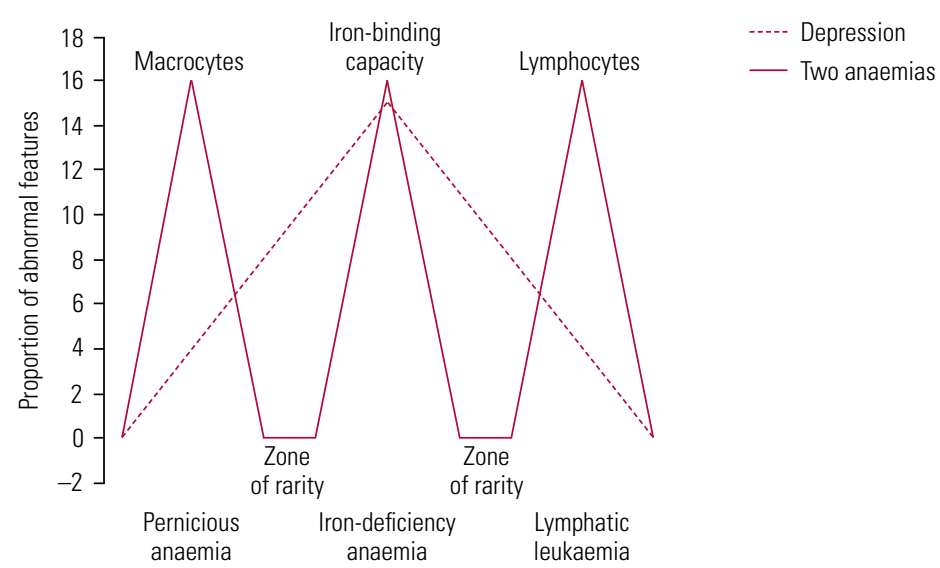

FIG 1

Schematic diagram showing the differences based on diagnostic tests between three common medical diagnoses, all of which cause anaemia (pernicious anaemia, irondeficiency anaemia and lymphatic leukaemia) and which can be detected by simple blood analysis, and the diagnosis of depression, which is based only on common symptoms. 
guidance in deciding on the cut-off point between disease and wellness. Almost all psychiatrists create an artificial boundary between disorder and normality. Increasingly, it has been recognised that a dimensional system of diagnosis is therefore superior to a categorical one, but this is only beginning to penetrate into diagnostic systems.

\section{The DSM and ICD classifications}

\section{Background}

The ICD is the official world classification. The section concerned with psychiatric disorders is called 'Mental and Behavioural Disorders'. This classification is used to record the diagnoses of all patients seen in psychiatric care across the world where official statistics are collected. By contrast, the DSM is the official classification in the USA for clinical diagnosis, although its influence now covers the globe, particularly because of its apparent advantages for research and the general belief that it is in some way 'more accurate'.

\section{Important dates}

The history of the official classification of disease began with two Frenchmen, Achille Guillard, who made the first attempt at a worldwide classification in 1853, and his grandson, Jacques Bertillon, Chief of Statistics for the City of Paris, who, under the auspices of the French government, convoked the first International Conference for the Revision of the International List of Causes of Death in Paris on 18 August 1900. This became the ICD and has since gone through ten revisions, with ICD-10 the latest to be published (World Health Organization 1992). The gap between ICD-10 and ICD-11 is likely to be 24 years, the longest between successive revisions since 1900. DSM has been revised more frequently, with successive revisions in 1987, 1994, 2000 and, more recently, in May 2013 (American Psychiatric Association 1987, 1994, 2000, 2013).

The DSM was first introduced in 1952 after the US military decided they needed to have a useful classification of mental disorders. It was not widely used initially, but with the arrival of Robert Spitzer, who coordinated the development of the third revision (DSM-III; American Psychiatric Association 1980), it became almost de rigeur to use the classification in research (Decker 2013) as, by comparison, the ICD equivalents were regarded as inadequate.

\section{Reliability}

The reason why DSM-III was a much better classification than any of its predecessors is because it used operational criteria (derived from psychiatric diagnostic criteria proposed 8 years earlier - the Feighner criteria; Feighner 1972). The insight given to Spitzer and colleagues (1975) by this earlier work was that psychiatric diagnosis could never be clinically valid or useful if it was unreliable, and the introduction of clear operational criteria in DSM-III undoubtedly improved reliability.

It is important to remember that the word 'reliable' in this context is a statistical concept, not in the general sense of being trustworthy and consistent. Statistical reliability is the extent to which one assessor will agree with another, and it is a self-evident truth that you will improve agreement between assessors when you give clear definitions of disorder rather than vague ones. Reliability includes both interrater reliability (assessments by different people at the same point in time) and temporal reliability (assessments carried out at different points in time). Good interrater and temporal reliability were the driving forces behind much of the work of the St Louis group of researchers under Samuel Guze in the 1960s and 1970s (Guze 1971, 1975), and these were the precursors of the Feighner criteria. These specific diagnostic criteria have dominated American psychiatric classification ever since.

By contrast, the ICD classification abhors clear diagnostic criteria unless they are independently validated, and allows the clinician to make judgements in the classification of disorder. Although this almost inevitably leads to less agreement in diagnosis, it should not automatically be regarded as less reliable (i.e. less accurate). It could be argued that good clinical judgement deserves to have a place in any classification what is the point of training and experience if it is not? - and so this is allowed much more space in the ICD classification than the DSM.

It is very important to realise that reliability in itself does not mean a disorder is better described or more valid in actually measuring what it purports to measure. It is always possible to get good agreement by having tight definitions of the condition concerned, but if it is not properly described its value will be equally limited. One obvious example of reliability in the absence of validity would be a survey of members of the Flat Earth Society answering the question 'Is the Earth flat or round?'. All would predict very high levels of agreement in a survey of this extreme population, probably a perfect correlation agreement of $r=1.0$ in favour of flatness, but this remarkable degree of reliability would not suddenly make the world less round. 
A similar example in psychiatry is the diagnosis of attention-deficit hyperactivity disorder (ADHD). In DSM-5, ADHD is characterised by a pattern of behaviour, present in multiple settings (e.g. at school, at home) that can result in 'performance issues' in social, educational or work settings. Symptoms are divided into two categories of inattention and hyperactivity/impulsivity including failure to pay close attention to details, difficulty organising tasks and activities, excessive talking, fidgeting or an inability to remain seated in appropriate situations - with a numerical score of symptoms given to set the threshold for diagnosis for the inattention, hyperactivity and impulsivity criteria.

It is perfectly possible to train a set of raters to achieve high degrees of reliability in making the diagnosis of ADHD, but this heterogeneous group of symptoms exhibited by a large number of children at some phase of development does not constitute a valid diagnosis. It also seems to share some of the uncertainty of roulette.

\section{Differences between DSM and ICD}

It is important to realise there is a lot of convergence between the two international systems of diagnosis, and that it is also possible to convert the diagnoses of one system into another. Although the main groups of psychiatric disorder are diagnosed similarly (Box 2), there are important differences with individual diagnoses in terms of both definition and name, and these differences are likely to increase with the introduction of ICD-11

BOX 2 Main subgroups of psychiatric disorder in both DSM and ICD

- Organic, including symptomatic, mental disorders

- Mental and behavioural disorders due to use of psychoactive substances

- Schizophrenia, schizotypal and delusional disorders

- Mood [affective] disorders

- Neurotic [a term now dropped], stress-related and somatoform disorders

- Behavioural syndromes associated with physiological disturbances and physical factors

- Disorders of personality and behaviour in adults

- Mental retardation

- Disorders of psychological development

- Behavioural and emotional disorders with onset usually occurring in childhood and adolescence

- Unspecified mental disorders
BOX 3 Main differences between ICD and DSM

\section{ICD}

- Intended for use by all health practitioners

- Special attention given to primary care and low- and middle-income countries

- Major focus on clinical utility (planned for ICD-11) with reduction of number of diagnoses

- Provides diagnostic descriptions and guidance but does not employ operational criteria
- Official world classification

\section{DSM}

- US classification (but used in many other countries)

- Used primarily by psychiatrists

- Focused mainly on secondary psychiatric care in high-income countries

- Tends to increase the number of diagnoses with each succeeding revision

- Diagnostic system depends on operational criteria using a polythetic system for most conditions (i.e. combination of criteria that need not all be the same)
(Box 3). This is also going to affect practice in the USA, as although it may not be fully appreciated by practitioners in the UK, every hospital diagnostic record has to use the ICD system, not the DSM one.

In discussing these differences, I must acknowledge a conflict of interest in that I am supporting the development of ICD-11 in various ways and have the personal view that it should replace the DSM system completely in the course of time - or, perhaps put more gently, that the DSM should ultimately merge with ICD. The main argument used by those who favour DSM is that it creates more accurate diagnosis. This may be true, partly because operational criteria are used but also because a much greater amount of resource and effort goes into making the classification than with ICD, which received very little funding indeed. As a consequence, ICD tends to follow palely in the footsteps of its big American brother, not wanting to be left behind but not quite sure what it is keeping up with.

\section{Validity}

As discussed earlier, reliability is no measure of validity, and although operational criteria may improve reliability, they can do so at the expense of validity. Thus, for example, a patient who satisfies all the criteria for major depression using the DSM system may have developed all the symptoms after a major event, not necessarily a traumatic one. The clinician may rightly conclude that the symptoms are a reaction to the event and not the onset of a depressive disorder requiring specific psychological or drug treatment. The clinician would therefore be thinking of this as an adjustment disorder, even though the operational criteria clearly marked this out as a major depressive disorder. This is 
MCO answers

$\begin{array}{lllll}1 b & 2 d & 3 e & 4 b & 5 c\end{array}$ not a textbook issue; in my clinical practice I have seen patients misdiagnosed and overtreated with antidepressants when it was quite clear that a wait-and-see approach would have been much more appropriate.

\section{Creation of more diagnoses/pathology}

The other problems associated with the preoccupation with reliability are a tendency to create more diagnoses rather than fewer, and also to create pathology out of normal variation. Allen Frances (2013), the former chair of the DSM-IV Task Force, has written persuasively about the dangers of this, and if clinical judgement is left aside in discussions over diagnosis, this tendency will only increase. The detection of pathology needs a scholarly overview and in the DSM compartmentalised system this is often lacking.

It is a sad fact that most of the diagnoses in both classification systems are seldom used, yet with each succeeding revision of DSM the number of diagnoses tends to increase (although there is a reduction of three diagnoses between DSM-IV and DSM-5), and each volume gets fatter.

\section{Who uses the two systems?}

DSM is mainly used by psychiatrists, although it is recognising that this is not entirely satisfactory and is promoting its use among psychologists and other mental health practitioners. ICD, on the other hand, has always had in mind 'the universal mental health practitioner', whether it be a barefoot doctor in Tanzania or a professor of psychiatry in Baltimore. It therefore has to be flexible and simple in the use of language to enable all practitioners - including many with very little formal qualifications in low- and middle-income countries - to be acceptable. One of the tasks of ICD is to show to practitioners that it can satisfy the critiques of diagnosis as spurious labelling and can serve practitioners and researchers well, but it has a long way to go in achieving these desired aims. This explains the key focus of ICD-11 clinical utility - as a diagnosis is only valuable if it can be used universally.

\section{Research Domain Criteria}

The DSM system will continue to be used because so much has been invested in it by the American Psychiatric Association and it is a well-resourced and diligent classification, and this remains true in spite of the abundant criticism that it has attracted. But it continues to be restrictive and has overreached itself, and the new US-initiated ambitious plan is to replace it by a new system of Research Domain Criteria (RDoC) that is linked more closely to neurobiological correlates (Cuthbert 2013).

The RDoC framework makes a clear conceptualisation of mental illnesses as brain disorders, but in contrast to neurological disorders where there are identifiable lesions, mental disorders are regarded as disorders of brain circuits. These circuit disorders can be tested by electrophysiology, functional neuroimaging and a range of new methods for quantifying connections in vivo. This process is complemented by data from genetics and clinical neuroscience to yield 'biosignatures' that add to clinical symptoms and signs and so improve clinical management.

This may be regarded as fanciful by some, but when one considers that we had no real understanding of mental functioning in vivo 20 years ago, a great deal of progress has been made since then. But it has not yet yielded results of note, and there are stern critics of this approach that cannot be dismissed lightly (Bracken 2012; Kleinman 2012).

\section{Conclusions}

Both DSM and ICD classification systems in psychiatry have value and, in particular, the high profile of DSM since 1980 has stimulated much more interest in nosology and heightened awareness of both the limitations and advantages of current classification.

DSM has been productive in promoting research, but has handicapped advances in some respects by giving credibility to diagnoses which probably do not exist (Markon 2013), and has generated much needless research into issues such as comorbidity of disorders which share much more than they differ by.

ICD has been poorly resourced and has not been able to generate the same degree of research data as DSM, but has steadily improved over the years and, with better descriptions and definitions, is likely to be used not only widely, but more seriously and accurately.

Diagnostic practice remains fluid and it is uncertain which of these three approaches - DSM, ICD or RDoC - will dominate in the end. None of these classification systems are going to be able to overcome the fundamental problems outlined at the beginning of this article until we have independent measures of disease, but for some mental conditions this will never happen. Without a well-functioning classification system we would be blind, deaf and stupid in the practice of our craft and so need to spring to its defence whenever it is mindlessly assaulted. 


\section{References}

American Psychiatric Association (1980) Diagnostic and Statistical Manual of Mental Disorders (3rd edn) (DSM-III). APA

American Psychiatric Association (1987) Diagnostic and Statistical Manual of Mental Disorders (3rd edn revised) (DSM-III-R). APA.

American Psychiatric Association (1994) Diagnostic and Statistical Manual of Mental Disorders (4th edn) (DSM-IV). APA.

American Psychiatric Association (2000) Diagnostic and Statistical Manual of Mental Disorders (4th edn, text revision) (DSM-IV-TR). APA.

American Psychiatric Association (2013) Diagnostic and Statistical Manual of Mental Disorders (5th edn) (DSM-5). APA.

Ayuso-Mateos JL, Nuevo R, Verdes E, et al (2010) From depressive symptoms to depressive disorders: the relevance of thresholds. British Journal of Psychiatry, 196: 365-71.

Bracken P, Thomas P, Timimi S, et al (2012) Psychiatry beyond the current paradigm. British Journal of Psychiatry, 201: 430-4.

Craddock N, Mynors-Wallis L (2014) Psychiatric diagnosis: impersonal, imperfect and important. British Journal of Psychiatry, 204: 93-5.

Cuthbert BN, Insel TR (2013) Toward the future of psychiatric diagnosis: the seven pillars of RDoC. BMC Medicine, 11: 126.

Dale RC, Heyman I, Giovannoni G, et al (2005) Incidence of anti-brain antibodies in children with obsessive-compulsive disorder. British Journal of Psychiatry, 187: 314-9.

Decker H (2013) The Making of DSM-III: A Diagnostic Manual's Conquest of American Psychiatry. Oxford University Press.
Feighner JP, Robins E, Guze SB, et al (1972) Diagnostic criteria for use in psychiatric research. Archives of General Psychiatry, 26: 57-63.

Frances A (2013) Saving Normal: An Insider's Revolt Against Out-ofControl Psychiatric Diagnosis, DSM-5, Big Pharma, and the Medicalization of Ordinary Life. William Morrow.

Guze SB, Goodwin DW (1971) Diagnostic consistency in antisocial personality. American Journal of Psychiatry, 128: 360-1.

Guze SB (1975) The validity and significance of the clinical diagnosis of hysteria (Briquet's syndrome). American Journal of Psychiatry, 132: 138-41.

Kendell RE (1968) The Classification of Depressive IIInesses (Institute of Psychiatry, Maudsley Monograph, No. 18). Oxford University Press.

Kleinman A (2012) Rebalancing academic psychiatry: why it needs to happen - and soon. British Journal of Psychiatry, 201: 421-2

Markon KE (2013) Epistemological pluralism and scientific development: an argument against authoritative nosologies. Journal of Personality Disorders, 27: 554-79.

Spitzer RL, Endicott J, Robins E (1975) Clinical criteria for psychiatric diagnosis and DSM-III. American Journal of Psychiatry, 132: 1187-92.

Timimi S (2011) Campaign to Abolish Psychiatric Diagnostic Systems such as ICD and DSM (CAPSID). Critical Psychiatry Network UK.

Tyrer P (2013) Models for Mental Disorder (5th edn). Wiley-Blackwell.

World Health Organization (1992) ICD-10: Classification of Mental and Behavioural Disorders. WHO.

\section{MCQs}

Select the single best option for each question stem

1 The DSM classification:

a is preferred by clinicians across the world

b shows better agreement between assessors than ICD

c is the most valid classification available

$\mathrm{d}$ is revised every 5 years

$\mathrm{e}$ is the standard classification for international data.

2 DSM and ICD classifications differ from other disease classifications in:

a being more widely used

b having clearer definitions

c being more appropriate for low- and middleincome countries d usually lacking biomarkers

e having many more diagnoses.

\section{ICD-11:}

a is likely to be very similar to DSM-5

b will have more diagnoses than ICD-10

c will not be used in North America

$\mathrm{d}$ will be the last revision of the ICD

$\mathrm{e}$ is focused on clinical utility.

4 Compared with DSM diagnoses, ICD

diagnoses:

a are interchangeable

b allow more clinical judgement in making diagnoses

c take longer to complete

$\mathrm{d}$ are more stable over time

e are preferred by researchers.
5 The main differences between ICD and DSM diagnoses in psychiatry are:

a ICD is more comprehensive than DSM

b DSM is more accurate than ICD

c ICD is the official international classification in psychiatry

d DSM is the only classification used in the USA

e ICD is used by more psychiatrists than DSM. 